\title{
OCORRÊNCIA DE HÍBRIDOS NÃO NATURAIS ENTRE BOTHROPS JARARACA E B. NEUWIEDI (SERPENTES, VIPERIDAE)
}

\author{
Rafael Lucchesi Balestrin ${ }^{1}$ \\ Moema Leitão-de-Araujo ${ }^{1}$ \\ Maria Lúcia Machado Alves ${ }^{1}$
}

\begin{abstract}
OCCURRENCE OF NON NATURAL HYBRIDISM BETWEEN BOTHROPS JARARACA AND $B$. NEUWIEDI (SERPENTES, VIPERIDAE). In May 1987, a female of Bothrops jararaca (Wied, 1824), from Carazinho, Rio Grande do Sul (RS), Brazil, was placed in the same vivarium with a male of Bothrops neuwiedi Wagler, 1824 coming from Guaíba, RS. There, they stayed for aproximately ten months. In March 1988, it was observed a delivery of five live and two still born, among them six presented morphologic characteristics of B. neuwiedi and one of B. jararaca. After the female died, in April 1988, through necropsy, two fetusus were found, one near the cloaca and, both identified as $B$. neuwiedi. The morphologic analysis and the origin of the progenitors suggest the hypothesis that the litter was resulted of cross-breeding.
\end{abstract}

KEYWORDS. Bothrops, hibridism, captivity, Rio Grande do Sul, Brazil.

\section{INTRODUÇÃO}

Casos de hibridismo na família Viperidae foram relatados por Cook (1955), que descreveu uma ninhada híbrida de Crotalus viridis Rafinesque, 1818 e C. scutulatus Kennicott, 1861, cuja cópula foi realizada em cativeiro e onde cada filhote híbrido apresentou características intermediárias entre os tipos paternais, com apenas um exemplar, do total de doze, apresentando padrão de colorido mais próximo a C. viridis. Para o gênero Bothrops Wagler, 1824, Vellard (1929) registrou um caso de hibridismo entre Bothrops jararaca (Wied,1824) e B. cotiara (Gomes, 1913); Prudente et al. (1995) entre B. alternatus Dumèril, Bibron \& Dumèril, 1854 e B. neuwiedi Wagler, 1824 proveniente da área da Usina Hidrelétrica de Segredo, Pinhão, Paraná, Brasil. Sobre a biologia reprodutiva, nascimento e desenvolvimento de $B$. jararaca, há registros de VANZoLINI (1946), Sazima (1988, 1989), Breno et al. (1990) e Alves et al. (2000).

Objetiva-se registrar a ocorrência de hibridismo não natural entre $B$. jararaca e $B$. neuwiedi em cativeiro, com descrição e análise das características de lepidose e biometria dos filhotes.

1.Núcleo Regional de Ofiologia de Porto Alegre, Museu de Ciências Naturais, Fundação Zoobotânica do Rio Grande do Sul, Caixa Postal 1188, 90001-970 Porto Alegre, RS, Brasil. (nopa@ffzb.org.br) 


\section{MATERIAL E MÉTODOS}

Foram observados uma fêmea de B. jararaca, coletada em 14 de maio de 1987 em Carazinho, Rio Grande do Sul (RS), Brasil (fig.1) e um macho de B. neuwiedi de Guaíba, RS, mantidos no mesmo viveiro, por carência de espaço físico no Núcleo Regional de Ofiologia de Porto Alegre (NOPA), Museu de Ciências Naturais (MCN), Fundação Zoobotânica do Rio Grande do Sul, desde a data da coleta até 03 de abril de 1988, quando do óbito da fêmea. Apesar de não ter sido observada cópula entre os exemplares, em 24 de março de 1988 a fêmea pariu cinco filhotes vivos e dois natimortos e, após sua morte, por necropsia, foram encontrados dois fetos. O total de nove exemplares foi incorporado à coleção do $\mathrm{MCN}$, a fêmea 9658 e os filhotes 9646 , 9647, 9659, 9665, 9679, 9693, 9714, 12320, 12321 (fig.2). O macho adulto não pôde ser preservado, pois foi encontrado em adiantado estado de decomposição, após final de semana prolongado.

Para identificação dos exemplares foram utilizadas as chaves dicotômicas de Peters \& OrejasMirAnda (1970) e Lema (1994), sendo também analisados os caracteres sistemáticos utilizados por CAMPBELL \& LAMAR (1989).

\section{RESULTADOS E DISCUSSÃO}

Dos nove filhotes, entre os quais dois natimortos e dois fetos, oito foram identificados como B. neuwiedi (tab.I), com a porção ventral clara ornamentada por numerosas manchas irregulares castanhas. A porção dorsal das cabeças apresenta o padrão característico de ornamentação da espécie, bem como uma linha postocular castanha. Dorsalmente, exibem coloração de fundo que varia em tons claros de marrom. Nos lados do corpo encontramse conjuntos de manchas escuras com formato trapezoidal, com pequenas máculas circulares próximas ou junto às arestas inferiores. Estes conjuntos se alternam nos lados do corpo e são intercalados por pequenas manchas castanhas circulares mais próximas da região vertebral (figs. 2, 3). A segunda escama supralabial não forma a margem anterior da fosseta loreal, característica diferencial entre as espécies em análise.

Apenas um filhote (MCN 9679) apresenta padrões cromáticos e ornamentais característicos de $B$. jararaca. Uma extensa área anômala na porção anterior da cabeça impossibilitou a observação de alguns dados de lepidose. Além da cabeça, a porção terminal da cauda se apresenta anômala e o espécime possui dimensões menores que as dos demais filhotes da ninhada (tab. 2, figs. 2, 4). Todos os exemplares da ninhada apresentam a ponta da cauda branca, característica comum em ambas as espécies (fig. 2).

Tabela I. Dados de lepidose dos exemplares híbridos não naturais entre Bothrops neuwiedi Wagler, 1824 e $B$. jararaca (Wied, 1824): A, anal; DO, dorsais; IL, infralabiais; IL/M, infralabiais em contato com mental anterior; IOC, interoculares; F, fêmea; M, macho; S, sexo; SC, subcaudais; SL, supralabiais; VE, ventrais;*dado não disponível.

\begin{tabular}{lrrrrrrrrr}
\hline MCN & 9646 & 9647 & 9659 & 9665 & 9679 & 9693 & 9714 & 12320 & 12321 \\
\hline DO & $23 / 23 / 21$ & $23 / 25 / 21$ & $21 / 23 / 19$ & $25 / 27 / 21$ & $23 / 25 / 19$ & $25 / 25 / 21$ & $23 / 23 / 21$ & $23 / 25 / 19$ & $23 / 23 / 19$ \\
VE & 182 & 175 & 177 & 176 & 174 & 180 & 182 & 178 & 179 \\
A & 1 & 1 & 1 & 1 & 1 & 1 & 1 & 1 & 1 \\
SC & $46 / 46$ & $55 / 55$ & $55 / 55$ & $43 / 43$ & $43 / 43$ & $44 / 44$ & $46 / 46$ & $53 / 53$ & $54 / 54$ \\
SL & $8 / 8$ & $8 / 8$ & $8 / 8$ & $8 / 8$ & $7 / 7$ & $8 / 9$ & $8 / 8$ & $7 / *$ & $8 / 8$ \\
IL & $11 / 11$ & $9 / 9$ & $11 / 10$ & $11 / 11$ & $11 / 10$ & $11 / 11$ & $12 / 12$ & $11 / *$ & $10 / 10$ \\
IL/M & $1-4 / 1-4$ & $1-3 / 1-3$ & $1-3 / 1-3$ & $1-4 / 1-4$ & $1-3 / 1-3$ & $1-4 / 1-4$ & $1-4 / 1-4$ & $1-4 / 1-4$ & $1-4 / 1-4$ \\
IOC & 10 & 9 & 9 & 10 & $*$ & 9 & 9 & 10 & 10 \\
S & $\mathrm{M}$ & $\mathrm{M}$ & $\mathrm{M}$ & $\mathrm{F}$ & $\mathrm{F}$ & $\mathrm{F}$ & $\mathrm{F}$ & $\mathrm{M}$ & $\mathrm{M}$ \\
\hline & & & & & & & & & \\
\hline
\end{tabular}



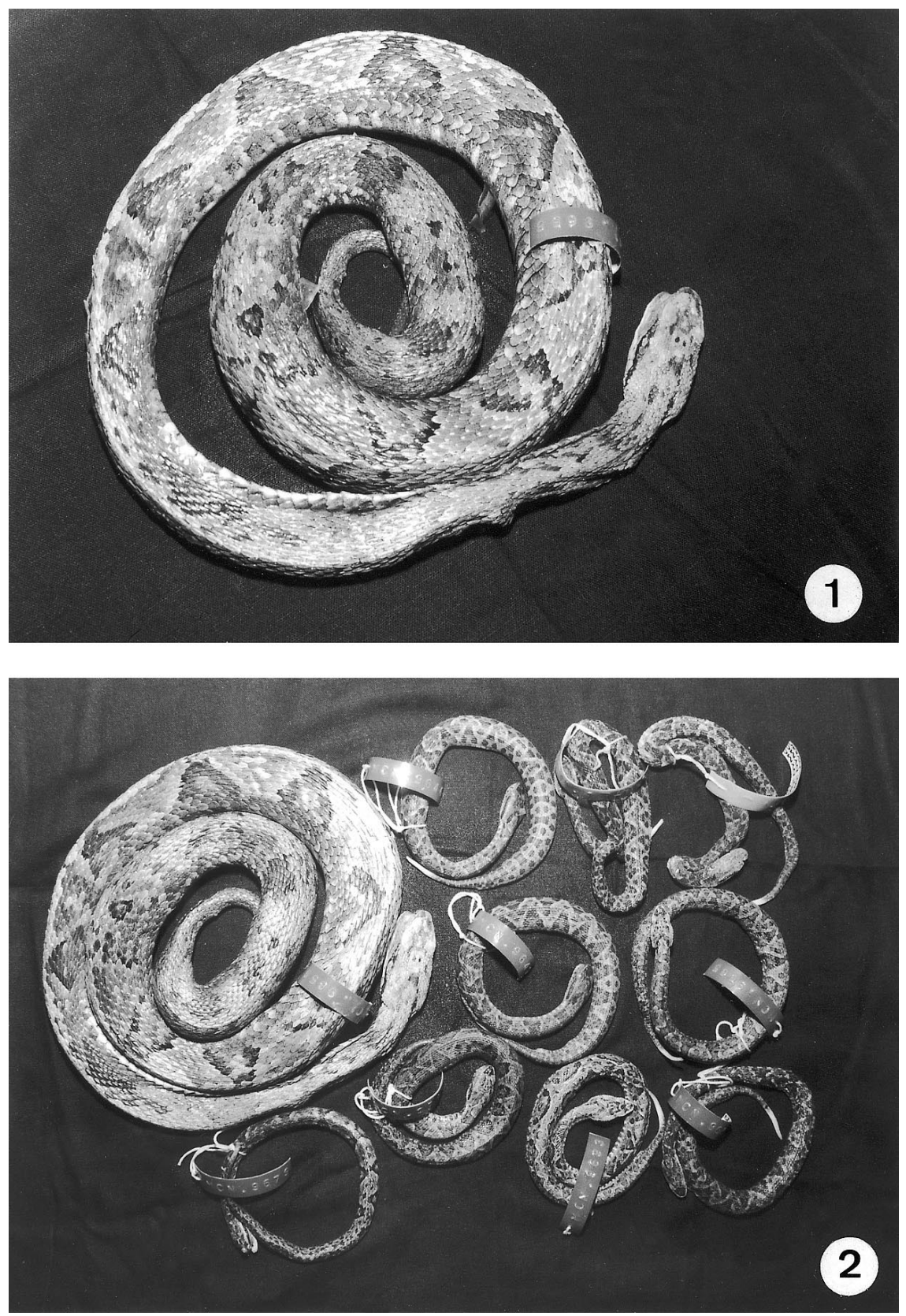

Figs. 1, 2. Bothrops jararaca (Wied, 1824), vista dorsal: 1, fêmea, progenitora de ninhada híbrida não natural; 2, fêmea e seus filhotes híbridos não naturais (fêmea, comprimento 1018,6 mm; filhotes, de 239,1 a 283,5 mm). 
Sendo oito dos nove filhotes identificados como B. neuwiedi, fica evidente a introgreção das características fenotípicas, como os desenhos dorsais e coloridos típicos de $B$. jararaca. Wilson Fernandes (com. pess.) informa que uma fêmea de B. neuwiedi, possível híbrido natural entre $B$. neuwiedi e $B$. jararaca, pariu 21 filhotes com padrões de desenho, ornamentação e dados merísticos de $B$. jararaca, introgredindo características fenotípicas de $B$. neuwiedi, indicando que os filhotes são resultantes de retrocruzamento $\operatorname{com} B$.jararaca. A progenitora apresentou dados merísticos compatíveis com B. neuwiedi, mas com um número de ventrais e subcaudais um pouco acima. Tais características, somadas a uma análise do eletroferograma do plasma e do número de organizadores nucleares (NORs) da fêmea, levou a crer ser ela um híbrido natural entre $B$. neuwiedi e $B$. jararaca e os filhotes produto do retrocruzamento com $B$. jararaca.

A lepidose da fêmea de B. jararaca (MCN 9658) é de 25/25/21 escamas dorsais, 184 ventrais, anal única, 50 subcaudais, 8/8 supralabiais, a segunda formando a margem anterior da fosseta loreal; $9 / 10$ infralabiais, 08 interoculares. $O$ comprimento total é de 1018,6 mm. Na impossibilidade de analisar as características de lepidose e biometria do macho, não pode ser afastada a hipótese de o mesmo ser um possível híbrido natural entre $B$. neuwiedi e $B$. jararaca, uma vez que ambas ocorrem na área de sua procedência, embora serem raros os registros de B. jararaca na coleção $\mathrm{MCN}$, para Guaíba. A progenitora da ninhada procede de Carazinho, região onde existe maior possibilidade de sintopia entre as espécies citadas. Apesar da fêmea apresentar um padrão externo característico de B.jararaca, ainda assim, pode ser um híbrido natural entre $B$. neuwiedi $\mathrm{e}$ B. jararaca, devido à introgreção de caracteres fenotípicos. Segundo W. Fernandes (com. pess.), que observou um macho adulto, procedente de Santa Catarina, Brasil, possível híbrido entre Bothrops alternatus e $B$. cotiara, com número de ventrais e subcaudais fora da gama da variação, mas com coloração, padrão de desenhos dorsais e hemipênis similares aos de B. cotiara. Acredita que tal hibridismo possa estar relacionado a drásticas alterações ambientais, possibilitando que espécies um dia segregadas passem a viver em sintopia.

PRUDENTE et al.(1995) registraram um possível híbrido natural entre B. alternatus e $B$. neuwiedi, que se assemelha a $B$. alternatus no padrão geral, mas apresenta a ponta da cauda branca e características hemipenianas semelhantes as de $B$. neuwiedi. Pondera que

Tabela II. Dados biométricos, em milímetros, dos exemplares híbridos não naturais entre Bothrops neuwiedi Wagler, 1824 e B. jararaca (Wied, 1824): AMA/CA, altura maior da cabeça; AME/CA, altura menor da cabeça; CA, comprimento da cabeça; $\mathrm{CT}$, comprimento total; CU, comprimento da cauda; LMA/CA, largura maior da cabeça; LME/CA, largura menor da cabeça; O/F, distância entre o olho e o frontal; O/NA, distância entre o olho e a narina; TR, comprimento do tronco; * dado não disponível.

\begin{tabular}{lrrrrrrrrr}
\hline MCN & 9646 & 9647 & 9659 & 9665 & 9679 & 9693 & 9714 & 12320 & 12321 \\
\hline CA & 17 & 16,4 & 17,2 & 17,4 & 15,1 & 17,5 & 17,2 & 17 & 17,3 \\
TR & 211 & 213 & 218 & 225 & 194 & 234 & 230 & 215 & 222 \\
CU & 32 & 36 & 39 & 39 & 30 & 32 & 33 & 38 & 37 \\
CT & 260 & 265,4 & 274,2 & 281,4 & 239,1 & 283,5 & 280,2 & 270 & 276,3 \\
O/NA & 3,55 & 3,65 & 3,6 & 3,85 & $*$ & 3,4 & 2,85 & $*$ & 3,65 \\
O/F & 5,6 & 5,2 & 5,1 & 5,35 & $*$ & 5,6 & 5,35 & $*$ & 5,95 \\
LMA/CA & 9,4 & 9,45 & 8,55 & 9,2 & $*$ & 10,3 & 9,3 & $*$ & 8,9 \\
LME/CA & 5,2 & 4,8 & 4,4 & 3,7 & $*$ & 4,75 & 3,4 & $*$ & 4,6 \\
AMA/CA & 6,6 & 6,15 & 6,55 & 6,85 & $*$ & 6,45 & 6,3 & $*$ & 5,35 \\
AME/CA & 4,45 & 4,25 & 4,2 & 4,45 & $*$ & 4,3 & 3,75 & $*$ & 3,65 \\
\hline
\end{tabular}



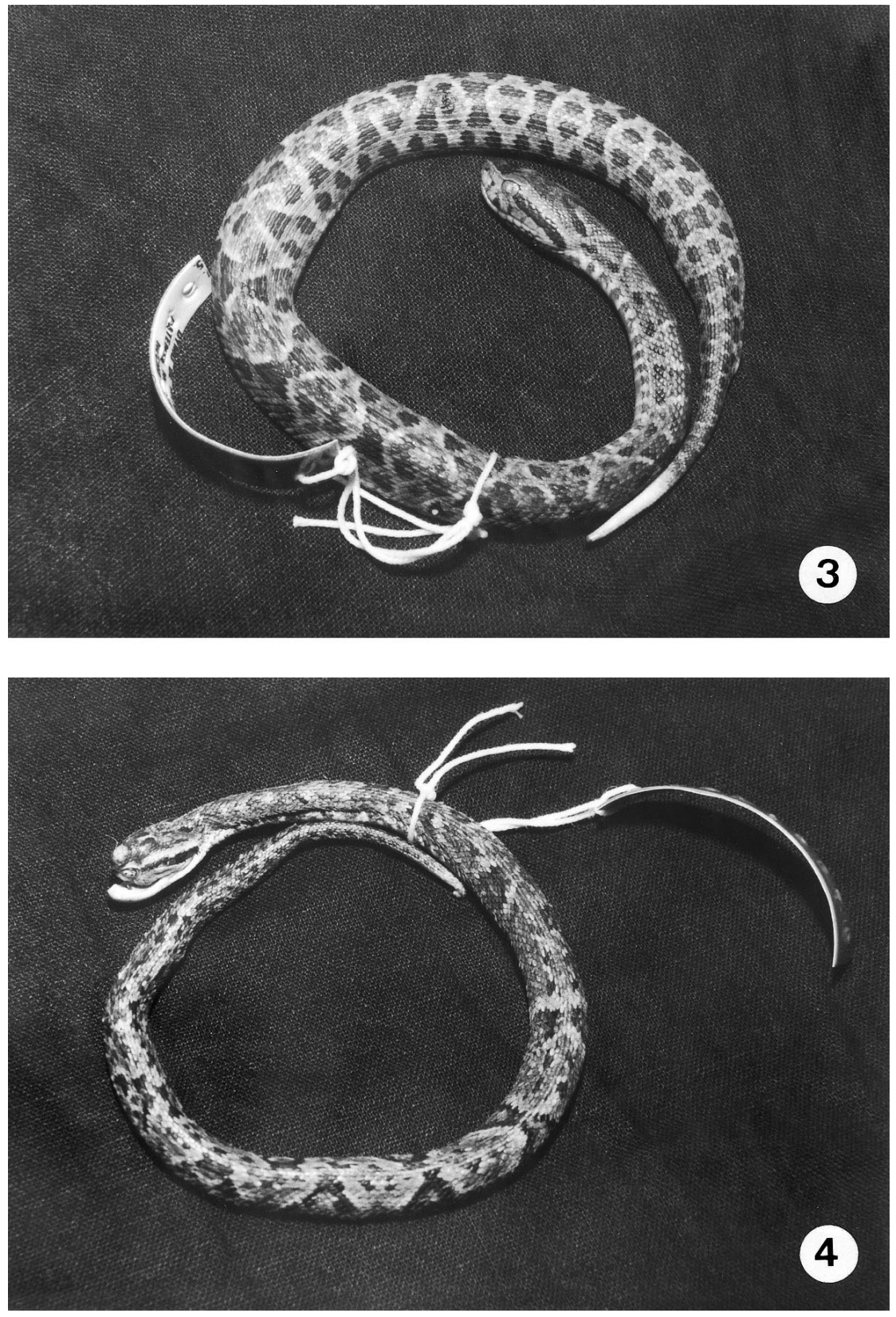

Figs. 3, 4. Híbrido não natural entre Bothrops jararaca (Wied, 1824) e B. neuwiedi Wagler, 1824, vista dorsal: 3, filhote com características fenotípicas de B. neuwiedi (comprimento $260 \mathrm{~mm}$ ); 4, filhote anômalo com características fenotípicas de B. jararaca (comprimento 239,1 mm). 
este caso não está relacionado a alterações ambientais, devido à significativa conservação da área da Usina Hidrelétrica de Segredo, Pinhão, Paraná, Brasil.

Como não foi observada cópula e considerando o tempo médio de gestação de $B$. neuwiedi, de 271 dias e de B. jararaca, entre 152 a 239 dias, conforme Alves et al. (1998, 2000), o período de observação, de 315 dias, não permite descartar a possibilidade de que a fêmea tenha chegado prenhe da natureza. Por menor que sejam as probabilidades, não se pode desconsiderar que a ninhada em estudo seja produto de um retrocruzamento, semelhante ao mencionado por W. Fernandes (com. pess.) entre B. neuwiedi e B. jararaca, provocado pela condição de cativeiro a que foram submetidos os exemplares.

\section{REFERÊNCIAS BIBLIOGRÁFICAS}

Alves, M. L. M.; Leitão-de-Araujo, M. \& Caberlon, E. 1998. Atividade reprodutiva de Bothrops neuwiedi em cativeiro (Serpentes, Viperidae). Iheringia, Sér. Zool., Porto Alegre, (84):185-191.

Alves, M. L. M.; Leitão-de-Araujo, M. \& Witt, A. A. 2000. Aspectos da biologia reprodutiva de Bothrops jararaca em cativeiro (Serpentes, Viperidae). Iheringia, Sér. Zool., Porto Alegre, (89):187-192.

Breno, M. C.; Yamanouye, N. et al. 1990. Maintenance of the snake Bothrops jararaca (Wied, 1824) in captivity. Snake, Nügata, 22:126-130.

Campbell, J. A. \& Lamar, W. W. 1989. The venemous reptiles of Latin America. New York, Comstock Publishing Associates. 425p.

CoOK, S. F., JR. 1955. Rattlesnake hybrids: Crotalus viridis x Crotalus scutulatus. Copeia, San Francisco, (2):139-141.

Lema, T. DE. 1994. Lista comentada dos répteis ocorrentes no Rio Grande do Sul, Brasil. Comun. Mus. Ciênc. Tecnologia PUCRS, Sér. Zool., Porto Alegre, 7:12-146.

Peters, J. A. \& Orejas-Miranda, B. 1970. Catalogue of the Neotropical Squamata. Part I Snakes. Bull. U. S. natn. Mus., Washington, (297):1-329.

Prudente, A. L. C.; Morato, S. A. A. \& Moura-Leite, J. C. De. 1995. Registro de um híbrido natural entre Bothrops alternatus Dumèril, Bibron \& Dumèril, 1854 e Bothrops neuwiedi Wagler, 1824 (Serpentes: Viperidae). Biociências, Porto Alegre, 3(2):231-238.

SAZIMA, I. 1988. Um estudo de biologia comportamental da jararaca Bothrops jararaca, com uso de marcas naturais. Mems Inst. Butantan, São Paulo, 50(3):83-99.

1989. Comportamento alimentar da jararaca, Bothrops jararaca: encontros provocados na natureza. Ciênc. Cult., São Paulo, 41(5):500-505.

VAnzolini, P. E. 1946. Regressão do pêso sobre o comprimento em Bothrops jararaca e sua variação sexual e estacional. Papéis Avuls Zool., São Paulo, 7(25):271-292.

Vellard, J. 1929. Un cas d'hybridisme chez les serpents Lachesis jararaca-cotiara. Bull. Soc. zool. Fr., Paris, 53:406-418.

Recebido em 27.07.2001; aceito em 07.01.2002. 